\section{Sleep duration and intensity of ADHD symptoms}

Rev. Bras. Psiquiatr. 2016;38:348-349

doi:10.1590/1516-4446-2015-1847

Attention deficit/hyperactivity disorder (ADHD) is one of the most common childhood psychiatric disorders. Its symptoms involve patterns of inattention, hyperactivity, and impulsivity, which are associated with global, social, academic, and adaptive impairment; need for treatment increases with each additional symptom. ${ }^{1}$

Children with ADHD commonly experience sleep problems, which add to difficulties in their daily life. ${ }^{2}$ Even in children with typical development, sleep deprivation can lead to behaviors similar to symptoms of ADHD, such as hyperactivity as a behavioral way of stabilizing vigilance ("brain arousal"), and cognitive deficits that can induce inattention. Although sleep duration appears not to be significantly different between children with typical development and those with $\mathrm{ADHD},{ }^{2}$ sleep duration might be associated with the intensity of ADHD behaviors, even in children with the disorder.

In this study, approved by the Ethics Committee of Universidade Federal de Minas Gerais, Brazil, 142 children with ADHD (116 boys and 26 girls; 42 inattentive, 10 hyperactive/impulsive, and 90 combined), aged 6 to 15 years (mean age $=9.42$ years, standard deviation $[S D]=$ 2.25) were enrolled. Caregivers completed the Brazilian version of the Schedule for Affective Disorders and Schizophrenia for School-Aged Children-Present and Lifetime Version (K-SADS-PL), ${ }^{3}$ and current inattentive (median $=8$, mean $=7.39, \mathrm{SD}=1.73$ ) and hyperactive-impulsive ( median $=7$, mean $=6.30, \mathrm{SD}=2.86$ ) symptoms were recorded. The sum of inattentive and hyperactive-impulsive symptoms can range from 0 to 9 for each dimension. Participants' sleep duration (time asleep at night, in hours) was computed as perceived by the caregiver (median $=10$, mean $=9.34, S D=1.40$ ), who reported the child's current time of sleep onset and current time of waking. Spearman's correlation showed a significant negative association between sleep duration and inattentive symptoms $\left(r_{(141)}=\right.$ -0.185, $p=0.027$ ), but not with hyperactive-impulsive symptoms $\left(r_{(141)}=0.101, \mathrm{p}=0.230\right)$. Participants with ADHD were then split by median inattentive symptoms, and the low-inattentive group ( $n=71$, median $=7$ symptoms) was shown to sleep more hours (median $=10$ hours, mean $=9.58, \mathrm{SD}=1.42)$ than the high-inattentive group $(\mathrm{n}=71$, median $=9$ symptoms; median $=9$ hours, mean $=9.10$, $\mathrm{SD}=1.34)(U=1.982, \mathrm{p}=0.024, r=-0.189)$.

Considering the complex origin of ADHD symptomatology and the knowledge that functional impairments are related to symptom intensity, ${ }^{1}$ even a small association should not be ignored. Inattentive symptoms seem to play a crucial and unique role in academic performance for children with $\mathrm{ADHD},{ }^{1}$ for example; thus, adequate investigation of sleep habits and ADHD symptomatology could be important not only to reduce ADHD severity but also to improve outcomes. Although we did not investigate the rate of sleep problems in our sample, sleep problems have been shown to be frequent and relevant to the management of children with $\mathrm{ADHD}{ }^{2}$ ADHD and sleep problems may share neurobiological pathways (cortical areas associated with regulation and arousal). However, these problems are thought to add functional impairments other than those caused by ADHD alone. ${ }^{4}$

This study has limitations that should be taken into account, such as the absence of medication control, lack of characterization of comorbid psychiatric disorders such as internalizing problems associated with insomnia, and lack of objective measures to determine sleep duration. Of note, although subjective and objective measures of sleep duration are moderately correlated, subjective measures (as used in this study) are usually biased. ${ }^{5}$

At any rate, our results suggest that sleep measures that are not commonly observed to differ between children with ADHD and children with typical development might still be useful for characterizing the role of sleep issues in ADHD severity. Most importantly, behavioral sleep interventions have demonstrated substantial and sustained benefits for improving sleep duration in children with $\mathrm{ADHD},{ }^{6}$ which highlights the potential benefit of sleep characterization in this population.

\section{Amanda P. Gomes-Tiago, ${ }^{1}$ Danielle de S. Costa, ${ }^{2}$ Antonio M. Alvim-Soares Jr., ${ }^{2}$ Leandro F. Malloy-Diniz,, ${ }^{3,5}$ Débora M. de Miranda ${ }^{4,5}$}

${ }^{1}$ Faculdade de Psicologia, Universidade Federal de Minas Gerais (UFMG), Belo Horizonte, MG, Brazil. ${ }^{2}$ Programa de Pós-Graduação em Medicina Molecular, UFMG, Belo Horizonte, MG, Brazil.

${ }^{3}$ Departamento de Psiquiatria, Faculdade de Medicina, UFMG, Belo Horizonte, MG, Brazil. ${ }^{4}$ Departamento de Pediatria, Faculdade de Medicina, UFMG, Belo Horizonte, MG, Brazil. ${ }^{5}$ Instituto Nacional de Ciência e Tecnologia - Medicina Molecular (INCT-MM),

Belo Horizonte, MG, Brazil.

Submitted Oct 27 2015, accepted Mar 212016.

\section{Acknowledgements}

The authors gratefully acknowledge all participants. This study was supported by the following Brazilian agencies through Instituto Nacional de Ciência e Tecnologia Medicina Molecular (INCT-MM): Conselho Nacional de Desenvolvimento Científico e Tecnológico (CNPq), Coordenação de Aperfeiçoamento de Pessoal de Nível Superior (CAPES), and Fundação de Amparo è Pesquisa do Estado de Minas Gerais (FAPEMIG; grant CBB-APQ-00075-09/ CNPq 573646/2008-2).

\section{Disclosure}

The authors report no conflicts of interest.

\section{References}

1 Lahey BB, Willcutt EG. Predictive validity of a continuous alternative to nominal subtypes of attention-deficit/hyperactivity disorder for DSM-V. J Clin Child Adolesc Psychol. 2010;39:761-75. 
2 Cortese S, Faraone SV, Konofal E, Lecendreux M. Sleep in children with attention-deficit/hyperactivity disorder: meta-analysis of subjective and objective studies. J Am Acad Child Adolesc Psychiatry. 2009;48: 894-908.

3 Brasil HHA. Desenvolvimento da versäo brasileira da K-SADS-PL (Schedule for Affective Disorders and Schizophrenia for Scholl Aged Children Present and Lifetime Version) e estudo de suas propriedades psicométricas [thesis]. São Paulo: Universidade Federal de São Paulo; 2003.

4 Cortese S, Brown TE, Corkum P, Gruber R, O'Brien LM, Stein M, et al. Assessment and management of sleep problems in youths with attention-deficit/hyperactivity disorder. J Am Acad Child Adolesc Psychiatry. 2013;52:784-96.

5 Lauderdale DS, Knutson KL, Yan LL, Liu K, Rathouz PJ. Sleep duration: how well do self-reports reflect objective measures? The CARDIA Sleep Study. Epidemiology. 2008;19:838-45.

6 Hiscock H, Sciberras E, Mensah F, Gerner B, Efron D, Khano S, et al. Impact of a behavioural sleep intervention on symptoms and sleep in children with attention deficit hyperactivity disorder, and parental mental health: randomised controlled trial. BMJ. 2015; 350:h68.

\section{Organic psychosis due to hypoparathyroidism in an older adult: a case report}

Rev. Bras. Psiquiatr. 2016;38:349-350

doi:10.1590/1516-4446-2015-1869

We present a case of psychosis secondary to iatrogenic hypoparathyroidism and hypocalcemia in a 73-year-old woman. At age 18, she had undergone a total thyroidectomy and developed severe psychotic symptoms due to unintended removal of the parathyroid glands, but had recovered fully after a hospital stay. For the last 15 years, she had been receiving periodic psychiatry follow-up for mild depressive and psychotic symptoms, consisting of auditory hallucinations (children's voices calling her "Mommy"). For years, she had been stable on a combination of fluoxetine $20 \mathrm{mg} /$ day and olanzapine $10 \mathrm{mg} /$ day. She had worked as a nurse with no functional impairment until retirement at age 65 .

The patient presented to the emergency department reporting anguish, auditory hallucinations of male voices that insulted her (which, according to the patient, came from electronic devices), and auditory misperceptions, with intolerance to electronic noises. The situation had worsened in the previous 6 months, and the patient now exhibited marked social withdrawal. She also presented a secondary depressed mood.

She was admitted to the psychiatric department. Initial treatment was olanzapine $10 \mathrm{mg} /$ day, with no therapeutic response. The patient crawled into the observation bay, stating the noise of the air conditioner was unbearable. Ten days after admission, olanzapine was stopped and risperidone $2 \mathrm{mg} /$ day and mirtazapine up to $30 \mathrm{mg} /$ day were introduced. Her condition improved, but she conti- nued to report hearing voices, although more bearable. She was discharged to the day hospital for diagnostic clarification.

The patient was found to have a mild ataxic gait worsened by extrapyramidal symptoms, with no clear improvement despite anticholinergic therapy. No other neurological signs of hypocalcemia were found. Blood tests showed hypocalcemia, hyperphosphatemia, and mild hypoparathyroidism (Table 1). A severe vitamin $D$ deficiency was also noticed (Table 1). We noticed that the patient had been refusing to take the calcium and calcitriol supplementation prescribed by her endocrinologist, leading to chronic hypocalcemia. Computed tomography scans showed bilateral calcifications in the globi pallidi and cerebellar dentate nuclei (Figure 1). Neuropsychological assessment showed mild cognitive impairment in some areas (attention/concentration, verbal initiative, and motor function).

Additional calcium supplementation was prescribed (calcium carbonate $4,000 \mathrm{mg}$ daily) and calcitriol was reintroduced ( $0.25 \mu \mathrm{g}$ twice daily), with subsequent improvement in laboratory values (Table 1). The patient's ataxic gait became barely perceptible and her auditory hallucinations, although persistent, became quieter and more bearable, with no interference with function or behavior. Accordingly, her secondary depressed mood improved to euthymia. Interestingly, motor function and attention, which were impaired at the first neuropsychological assessment, also improved after calcium and calcitriol supplementation. Symptomatic treatment of psychosis with risperidone $2 \mathrm{mg} /$ day was reduced to $1 \mathrm{mg} /$ day and later switched to paliperidone $3 \mathrm{mg} /$ day due to intolerable extrapyramidal symptoms. No worsening was noticed after the dose reduction and switch in antipsychotic agent.

Few cases of hypoparathyroidism and hypocalcemia with neuropsychiatric manifestations have been reported. ${ }^{1,2}$ Basal ganglia calcification involving the globus pallidus and cerebellum is typical, perhaps due to hyper-

Table 1 Blood test results at admission and after 6 months of calcium and vitamin D supplementation

\begin{tabular}{|c|c|c|c|}
\hline & \multicolumn{2}{|c|}{ Value } & \multirow[b]{2}{*}{ Normal range } \\
\hline & Admission & Follow-up & \\
\hline Ionized calcium (mmol/L) & 0.84 & 1.24 & $1.13-1.32$ \\
\hline Phosphorus (mmol/L) & 5.40 & 4.3 & $2.70-4.5$ \\
\hline Magnesium (mmol/L) & 0.68 & 0.80 & $0.78-1.02$ \\
\hline Parathyroid hormone $(\mathrm{pg} / \mathrm{mL})$ & 8.40 & 9.40 & $10-55$ \\
\hline Vitamin $\mathrm{D}(\mathrm{ng} / \mathrm{mL})$ & 13 & 27 & $>30$ \\
\hline
\end{tabular}

